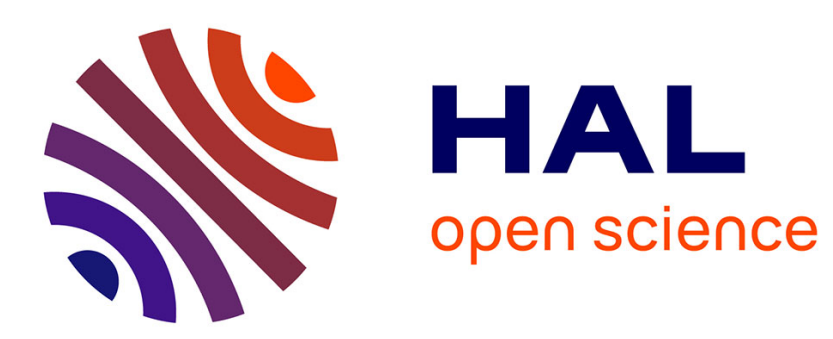

\title{
HMBC-like experiment based on longitudinal csa/dipolar cross-correlation
}

Sabine Bouguet-Bonnet, Sebastien Leclerc, Pierre Mutzenhardt, Daniel Canet

\section{To cite this version:}

Sabine Bouguet-Bonnet, Sebastien Leclerc, Pierre Mutzenhardt, Daniel Canet. HMBC-like experiment based on longitudinal csa/dipolar cross-correlation. Journal of Magnetic Resonance, 2005, 173 (1), pp.29-33. 10.1016/j.jmr.2004.11.012 . hal-00125370

\section{HAL Id: hal-00125370 https://hal.science/hal-00125370}

Submitted on 8 Mar 2017

HAL is a multi-disciplinary open access archive for the deposit and dissemination of scientific research documents, whether they are published or not. The documents may come from teaching and research institutions in France or abroad, or from public or private research centers.
L'archive ouverte pluridisciplinaire HAL, est destinée au dépôt et à la diffusion de documents scientifiques de niveau recherche, publiés ou non, émanant des établissements d'enseignement et de recherche français ou étrangers, des laboratoires publics ou privés. 


\section{HMBC-like experiment based on longitudinal csa/dipolar cross-correlation.}

Sabine Bouguet-Bonnet, Sébastien Leclerc, Pierre Mutzenhardt, Daniel Canet ${ }^{*}$

Laboratoire de Méthodologie RMN

Université Henri Poincaré

Faculté des Sciences

BP 239

54506 Vandoeuvre-lès-Nancy Cedex

France 


\begin{abstract}
A gradient-based sequence is proposed for efficiently filtering out all quantities except the longitudinal two-spin order, created by csa/dipolar cross-correlation rates. The dipolar interaction is between a proton and a heteronucleus, the csa being generally the one of the heteronucleus. Proton detection is carried out in order to benefit from maximum sensitivity. The resulting two-dimensional spectrum has the same aspect as an HMBC spectrum, implying the existence of a $J$ coupling between the two considered nuclei, but here cross-peaks indicate the strength of the relevant csa/dipolar cross-correlation rate. The method is especially interesting in the case of medium-sized molecules where a given heteronucleus is subjected to several csa/dipolar cross-correlation effects, along with the corresponding $J$ couplings.
\end{abstract}

Key Words: cross-correlation, multidimensional NMR, NMR, relaxation. 
Interference between the chemical shift anisotropy (csa) relaxation mechanism and the dipolar relaxation mechanism (generally between a proton and a heteronucleus, the csa concerning, most of the time, the heteronucleus denoted as $\mathrm{X}$ in the following) has been known for several decades $[1,2]$. The interest of this cross-correlation relaxation parameter lies in its dependency with respect to structural and dynamical properties. It is nowadays widely exploited, in particular for studying biological macromolecular systems $[3,4,5,6,7$, $8,9,10,11,12]$. The simplest way to exploit this feature is to rely upon the transverse crosscorrelation rate, which manifests itself by different transverse relaxation rates of the two components in the $\mathrm{X}$ doublet of splitting $J_{X H}$ [2]. We address here the problem of longitudinal csa-dipolar cross-correlation which is less straightforward to measure, although several efficient methods exist for small molecules and also for biological macromolecules $[10,11$, $13,14,15,16,17,18,19]$. Our goal here is to sort out the different cross-correlation effects which possibly exist and which can be detected provided that the considered heteronucleus is $J$-coupled to several protons. Although of general applicability, the method that we are going to describe concerns preferentially small or medium-sized molecules where several ${ }^{n} J_{X H}$ couplings can be resolved. Owing to the fact that the $r_{X H}$ distance is involved into crosscorrelation rates by $1 / r_{X H}^{3}$ (and not $1 / r_{X H}^{6}$ as in auto or cross-relaxation rates), such measurements should be feasible even for relatively remote protons.

The proposed two-dimensional experiment is derived from a one-dimensional method previously presented [20]. The sequence is depicted in figure 1. It starts with transverse carbon-13 magnetization which is labelled only by carbon chemical shifts during the evolution time $t_{1}$. The next $\pi / 2$ pulse takes the labelled magnetization towards $-\mathrm{z}$, all residual transverse magnetization being suppressed by the first gradient pulse $\left(g_{1}\right)$. Then comes the mixing time, during which relaxation occurs because carbon magnetization is off-equilibrium. Among all relaxation processes, only cross-correlation is able to create a longitudinal spin 
order (represented by $2 I_{Z}^{C} I_{Z}^{H}$ ) and only the latter is preserved with the filter described in the following. At the outcome of the mixing time, a carbon-13 $\pi / 2$ pulse transforms the longitudinal order into $2 I_{Y}^{C} I_{Z}^{H}$. The bipolar gradient pulses $\mathrm{g}_{2}$ are assumed to produce a dephasing by an angle $\theta$ at a given location so that one has :

$$
2 I_{Y}^{C} I_{Z}^{H} \cos \theta+2 I_{X}^{C} I_{Z}^{H} \sin \theta
$$

The two subsequent $(\pi / 2)$ pulses lead to :

$$
-2 I_{Z}^{C} I_{Y}^{H} \cos \theta+2 I_{X}^{C} I_{Y}^{H} \sin \theta
$$

The amplitude of the $\mathrm{g}_{3}$ gradients $\left(\mathrm{g}_{3}=\mathrm{g}_{2} / 4\right)$ are such that they produce again a dephasing of $\theta$ and at the end we have:

$$
-2 I_{Z}^{C} I_{Y}^{H} \cos ^{2} \theta-2 I_{Z}^{C} I_{X}^{H} \cos \theta \sin \theta+2 I_{X}^{C} I_{Y}^{H} \cos \theta \sin \theta+2 I_{X}^{C} I_{X}^{H} \cos ^{2} \theta
$$

Owing to the space average $\left(\left\langle\cos ^{2} \theta\right\rangle=\left\langle\sin ^{2} \theta\right\rangle=1 / 2,\langle\sin \theta \cos \theta\rangle=0\right)$, the quantities which survive can be written as:

$$
-\frac{1}{2}\left(2 I_{Z}^{C} I_{Y}^{H}\right)+\frac{1}{2}\left(2 I_{X}^{C} I_{X}^{H}\right)
$$

The second quantity being unobservable, and all other coherences being eliminated by the gradient pulses, one is left with an antiphase proton doublet (provided that a relevant $J$ coupling exists), the intensity of which reflects the amplitude of the longitudinal spin order before the filter, thus the effect of csa/dipolar cross-correlation.

The method has been checked with a medium size molecule. We have chosen a symmetric dioxole (sketched in figure 2 and denoted nd11 in the following) dissolved in DMSO- $\mathrm{d}_{6}$ which has been previously studied in this laboratory and for which spin relaxation, dynamics and carbon shielding tensors have been fully characterized [21, 22]. From these data, all $\operatorname{csa}\left({ }^{13} \mathrm{C}\right) / \operatorname{dipolar}(\mathrm{CH})$ cross correlation rates have been calculated according to the following expression: 


$$
\eta_{z}=-\frac{1}{5}\left(\frac{\mu_{0}}{4 \pi}\right)\left(\frac{\hbar \gamma_{H} \gamma_{N}}{r_{C H}^{3}}\right) \omega_{C} \Delta \sigma \widetilde{J}\left(\omega_{C}\right)
$$

$\Delta \sigma$ being the ${ }^{13} \mathrm{C}$ chemical shift anisotropy, and $r_{C H}$ the distance between the considered carbon and proton. $\widetilde{J}\left(\omega_{C}\right)$ are normalized spectral densities which depend solely on dynamical parameters and which account for a possible reorientation anisotropy. Significant cross correlation rates are reported in table 1.

Experiments were carried out at ambient temperature with a Bruker Avance DRX spectrometer operating at 14.1 Tesla $\left(600 \mathrm{MHz}\right.$ and $150 \mathrm{MHz}$ for ${ }^{1} \mathrm{H}$ and ${ }^{13} \mathrm{C}$, respectively). The results are displayed in figure 3 . Owing to the fact that carbon-13 is at the natural abundance level, one can notice especially clear spectra and also the ability to detect weak cross-peaks (see the insert of figure 3). This spectral quality is further enhanced by the possibility of recording pure absorption spectra. However, as far as a single mixing time is considered, these results in terms of signal intensities are qualitative, due to the fact that the magnetization build-up according to cross-correlation can significantly vary from one $\mathrm{C}-\mathrm{H}$ pair to the other. Nevertheless, the interest of such a spectrum can be appreciated by comparison with the more classical HMBC [23] spectrum (figure 4). It can be seen that between spectra of figures 3 and 4, we observe qualitatively the same type of information, that is correlations arising from heteronuclear $J$ couplings. Cross peak intensities in HMBC spectra are essentially governed by the value of the remote $J$ couplings; conversely, in our experiment, intensities are governed by the csa/dipolar cross-correlation rates. This feature is especially attractive for the chosen molecule for which we were able to detect correlations between symmetrically equivalent sites (see for instance the $\left(6,6^{\prime}\right)$ pair).

It is possible to understand the cross-peak intensities or the lack of cross-peaks according to the following considerations. i) For observing a cross-peak, the corresponding cross correlation rate value, as given in table 1, must be large enough (typically greater than $0.310^{-}$ 
${ }^{2} \mathrm{~s}^{-1}$ ), ii) due to the antiphase character of the response, the involved ${ }^{\mathrm{n}} J_{\mathrm{CH}}$ coupling must be large enough for avoiding mutual cancellation of the two lines (in phase and antiphase) in the considered multiplet. These two features explain all the results, owing to the fact that ${ }^{2} J_{\mathrm{CH}}$ couplings are usually weak in aromatic systems (of the order of $1 \mathrm{~Hz}$ ) whereas ${ }^{3} J_{\mathrm{CH}}$ are sufficiently large (of the order of $4 \mathrm{~Hz}$ for a cis configuration and $7 \mathrm{~Hz}$ for a trans configuration). Because ${ }^{1} J_{\mathrm{CH}}$, as well as the corresponding cross-correlation rate are large, all one-bond correlations are obviously visible. In order to illustrate the above considerations, we can notice the lack of correlations between $\mathrm{C}_{3}$ and $\mathrm{H}_{5}$ and $\mathrm{H}_{5}$, (the two responses being expected at the same location). This is easily explained by i) the weak value of the crosscorrelation rate for $\mathrm{H}_{5}$ even if a three bond coupling is involved, ii) weak values for both cross-correlation rate and the $J$ coupling for $H_{5}$, On the other hand, the surprisingly weak $\mathrm{C}_{6^{-}}$ $\mathrm{H}_{5}, \mathrm{H}_{5}$, response can be explained by the fact that the cross correlation rates are of opposite sign (see table 1) and, therefore, tend to cancel each other.

The essential merit of the present experiment is to display all csa-dipolar cross-correlation in a single measurement and in a semi-quantitative fashion. Conversely, for sensitivity reasons, it is difficult to consider this experiment for effectively measuring cross-correlation rates. In fact, any attempt to enhance the sensitivity by initially transferring the proton polarization toward carbon-13 by means of $J$ would fail because of the magnitude and the variety of these multiple bond couplings. Nevertheless, if one really wishes to measure accurately a csa-dipolar cross-correlation rate, one can always focus, from these two dimensional results, on a given $\mathrm{CH}$ pair (thus with a given $J$ value) and derive from the present experiment a selective one-dimensional quantitative procedure (see ref [20]). 


\section{References}

[1] E.L. Mackor, C. MacLean, Relaxation processes in systems of two non-identical spins, Progr. NMR Spectrosc. 3 (1967) 129-157.

[2] M. Goldman, Interference effects in the relaxation of a pair of unlike spin- nuclei, J.Magn.Reson. 60 (1984) 437-452.

[3] N. Tjandra , A. Szabo, A. Bax, Protein Backbone Dynamics and ${ }^{15} \mathrm{~N}$ Chemical Shift Anisotropy from Quantitative Measurement of Relaxation Interference Effects, J. Am. Chem. Soc. 118 (1996) 6986-6991.

[4] M. Tessari, H. Vis, R. Boelens, R. Kaptein R., G.W. Vuister, Quantitative Measurement of Relaxation Interference Effects between ${ }^{1} \mathrm{H}^{\mathrm{N}}$ csa and ${ }^{1} \mathrm{H}-{ }^{15} \mathrm{~N}$ Dipolar Interaction: Correlation with Secondary Structure, J.Am.Chem.Soc. 119 (1997) 8985-8990.

[5] M. Tessari, F.A.A. Mulder, R. Boelens, G.W. Vuister, Determination of Amide Proton CSA in $15 \mathrm{~N}$-Labeled Proteins Using ${ }^{1} \mathrm{H}$ csa $/{ }^{15} \mathrm{~N}-{ }^{1} \mathrm{H}$ Dipolar and ${ }^{15} \mathrm{~N}$ CSA $/{ }^{15} \mathrm{~N}-{ }^{1} \mathrm{H}$ Dipolar Cross-Correlation Rates, J.Magn.Reson. 127 (1997) 128-133.

[6] N. Tjandra, A. Bax, Large Variations in ${ }^{13} \mathrm{C}^{\alpha}$ Chemical Shift Anisotropy in Proteins Correlate with Secondary Structure, J.Am.Chem.Soc. 119 (1997) 9576-9577.

[7] N. Tjandra, A. Bax, Measurement of amide proton chemical shift anisotropy in ${ }^{15} \mathrm{~N}$ enriched proteins. Correlation with hydrogen bond length, J.Am.Chem.Soc. 119 (1997) 80768082.

[8] D. Fushman, N. Tjandra, D. Cowburn, Direct measurement of ${ }^{15} \mathrm{~N}$ chemical shift anisotropy in solution, J.Am.Chem.Soc. 120 (1998) 10947-10952.

[9] D. Fushman, D. Cowburn, Model-independent analysis of ${ }^{15} \mathrm{~N}$ chemical shift anisotropy from NMR relaxation data. Ubiquitin as a test example, J.Am.Chem.Soc. 120 (1998), 71097110. 
[10] C.D. Kroenke, J.P. Loria, L.K.Lee, M.Rance, A.G. Palmer III, Longitudinal and transverse $1 \mathrm{H}-15 \mathrm{~N}$ dipolar/15N chemical shift anisotropy relaxation interference : unambiguous determination of rotational diffusion tensor and chemical exchange effects in biological macromolecules, J. Am. Chem. Soc. 120 (1998) 7905-7915.

[11] P. Damberg, J. Jarvet, P. Allard, A. Graslund, Quantitative estimation of magnitude and orientation of the csa tensor from field dependence of longitudinal NMR relaxation rates, J.Biomol.NMR 15 (1999) 27-37.

[12] J.B. Hall, D. Fushman, Characterization of the overall and local dynamics of a protein with intermediate rotational anisotropy : differentiating between conformational exchange and anisotropic diffusion in the B3 domain of protein G, J. Biomol. NMR 27 (2003) 261-275.

[13] J.B. Hall, D. Fushman, Direct measurement of the transverse and longitudinal ${ }^{15} \mathrm{~N}$ chemical shift anisotropy-dipolar cross-correlation rate constants using ${ }^{1} \mathrm{H}$-coupled HSQC spectra, Magn. Reson. Chem. 41 (2003) 837-842.

[14] L. Wang, A.V. Kurochkin, E.R.P. Zuiderweg, An iterative fitting procedure for the determination of longitudinal NMR cross-correlation rates, J. Magn. Reson. 144 (2000) 175185.

[15] K.E. Köver, G. Batta, Separating structure and dynamics in csa/dd cross-correlated relaxation : a case study on trehalose and ubiquitin, J. Magn. Reson. 150 (2001) 137-146.

[16] H. Néry, D. Canet, F. Toma, S. Fermandjian, Carbon-13 nuclear magnetic relaxation in ${ }^{13} \mathrm{C}$ uniformly enriched glycine and aspartic acid, J. Am. Chem. Soc. 105 (1983) 1482-1486. [17] G. Jaccard, S. Wimperis, G. Bodenhausen, Observation of $2 \mathrm{I}_{\mathrm{z}} \mathrm{S}_{\mathrm{z}}$ order in NMR relaxation studies for measuring cross-correlation of chemical shift anisotropy and dipolar interactions, Chem. Phys. Lett. 138 (1987) 601-606. 
[18] N. Müller, G. Bodenhausen, Cross correlation of chemical shift anisotropy and dipolar interactions in methyl protons investigated by selective nuclear magnetic resonance spectroscopy, J. Chem. Phys. 98 (1993) 6062-6069.

[19 ] G. Batta, K.E. Köver, J. Kowalewski, A comparison of 1D and 2D (unbiased) experimental methods for measuring csa/dd cross-correlated relaxation, J. Magn. Reson. 136 (1999) 37-46.

[20] S. Leclerc, S. Bouguet-Bonnet, P. Mutzenhardt, J. Brondeau, D. Canet, Simulation of radio-frequency field inhomogeneity effects: application to pulse trains aimed at the determination of csa-dipolar interference terms, Magn. Reson. Chem. 41 (2003) 769-775.

[21] O. Walker, P. Mutzenhardt, P. Tekely, D. Canet, Determination of carbon-13 chemical shielding tensor in the liquid state by combining NMR relaxation experiments and quantumchemical calculations, J. Am. Chem. Soc. 124 (2002) 865-873.

[22] O. Walker, P. Mutzenhardt, D. Canet, Heteronuclear Overhauser experiments for symmetric molecules, Magn. Reson. Chem 41 (2003) 776-781.

[23] A. Bax, M.F. Summers, ${ }^{1} \mathrm{H}$ and ${ }^{13} \mathrm{C}$ assignments from sensitivity enhanced detection of heteronuclear multiple-bond connectivity by two-dimensional multiple quantum NMR, J. Am. Chem. Soc. 108 (1986) 2093-2094. 


\section{Table and Figure captions}

Table 1

Values (in $10^{-2} \mathrm{~s}^{-1}$ ) of csa/dipolar cross correlation rates for nd11 calculated as explained in the text, with structural and dynamical parameters published by Walker et al. [21, 22], and for a magnetic field of $14.1 \mathrm{~T}$. They have been limited to those which have a value greater than 0.1 $10^{-2} \mathrm{~s}^{-1}$. Stars indicate experimentally undetected cross-correlation. The number of bonds separating the considered carbon and proton(s) is indicated in the upper left corner of each cell.

\begin{tabular}{|c|c|c|c|c|c|c|c|}
\hline${ }^{1}$ & 1 & 3 & 3' & 5 & $5^{\prime}$ & 6 & $6^{\prime}$ \\
\hline 1 & $\begin{array}{l}1 \\
5.68\end{array}$ & & & & & & \\
\hline 2 & $\begin{array}{ll}3 & * \\
0.28\end{array}$ & $\begin{array}{l}2 \\
-0.81\end{array}$ & $\begin{array}{l}3 \\
0.40\end{array}$ & & & & \\
\hline 3 & & $\begin{array}{l}1 \\
13.87\end{array}$ & $\begin{array}{l}4 \quad{ }^{*} \\
0.30\end{array}$ & $3{ }^{*}{ }^{*}{ }^{2}$ & $\begin{array}{c}4 \\
0.23\end{array}$ & & \\
\hline 4 & & 2.11 & $\begin{array}{l}3 \\
0.30\end{array}$ & ${ }^{2} 1.56$ & ${ }^{3} 0.42$ & 3 & $\begin{array}{l}4 \\
0.52\end{array}$ \\
\hline 5 & & $\begin{array}{lc}3 & * \\
-0.05\end{array}$ & $\begin{array}{l}4 \\
0.29\end{array}$ & $\begin{array}{l}1 \\
24.98\end{array}$ & $\begin{array}{c}4 \\
0.55\end{array}$ & $\begin{array}{l}2 \\
-0.24\end{array}$ & 3 \\
\hline 6 & & & & $\begin{array}{l}2 \\
-0.82\end{array}$ & $\begin{array}{l}3 \\
0.57\end{array}$ & $\begin{array}{l}1 \\
39.34\end{array}$ & $\begin{array}{l}2 \\
-0.89\end{array}$ \\
\hline
\end{tabular}




\section{Figure 1}

The sequence used to produce $2 \mathrm{D}$ spectra that contain exclusively antiphase doublets arising from csa/dipolar cross-correlation effects. The first gradient pulse $\left(g_{1}\right)$ destroys any residual transverse magnetization at the beginning of the mixing time. The filter is based on the bipolar gradients $g_{2}$ and $g_{3}$ (their $\pi$ pulses ensure that the $g_{2}$ pulses act only on carbon-13 while $g_{3}$ pulses act only on proton). The ratio of gradient strengths $g_{2} / g_{3}$ is equal to $\gamma_{H} / \gamma_{C}$. The final spin-lock pulse $\left((\mathrm{SL})_{\mathrm{y}}\right.$, duration $\left.1 \mathrm{~ms}\right)$ and the simple phase cycle $\left((\pi / 2)_{ \pm x} ;\right.$ acq \pm$)$ help to eliminate unwanted residual coherences. A States-TPPI modulation was used. To enhance the initial ${ }^{13} \mathrm{C}$ polarization by nOe, proton decoupling can be applied during the recycle time.

\section{Figure 2}

The molecule of 2,3-naphto-1,3-dioxole chosen for its symmetry properties which further illustrate the capabilities of the method described in this paper.

\section{Figure 3}

The $2 \mathrm{D}$ spectrum $(1024 \times 512$ data points $)$ resulting from the sequence of figure 1 applied to the molecule of figure 2 at a field of 14.1Tesla. 64 scans were accumulated for each $t_{1}$ increment (128 increments of $36 \mu \mathrm{s})$. A mixing time of $1 \mathrm{~s}$ and a repetition time of 10 s were used. Peaks and the locations in the molecule of figure 2 are numbered accordingly (the prime for symmetric sites has been omitted). In the insert is displayed a representative proton crosssection (at the level of the $\mathrm{C}_{4}$ chemical shift). 
Figure 4

HMBC spectrum (at 14.1T) of the molecule of figure 2. 8 scans were accumulated for each $t_{1}$ increment (256 $t_{1}$ values with $50 \mu$ s increments). The interval for the evolution according to long-range $J$ couplings was set at $77 \mathrm{~ms}$. The repetition time was set at $2.5 \mathrm{~s}$. For comparison purpose with figure 3, the initial subsequence used for canceling one-bond correlations (via $\left.{ }^{1} J_{C H}\right)$ has been omitted. 


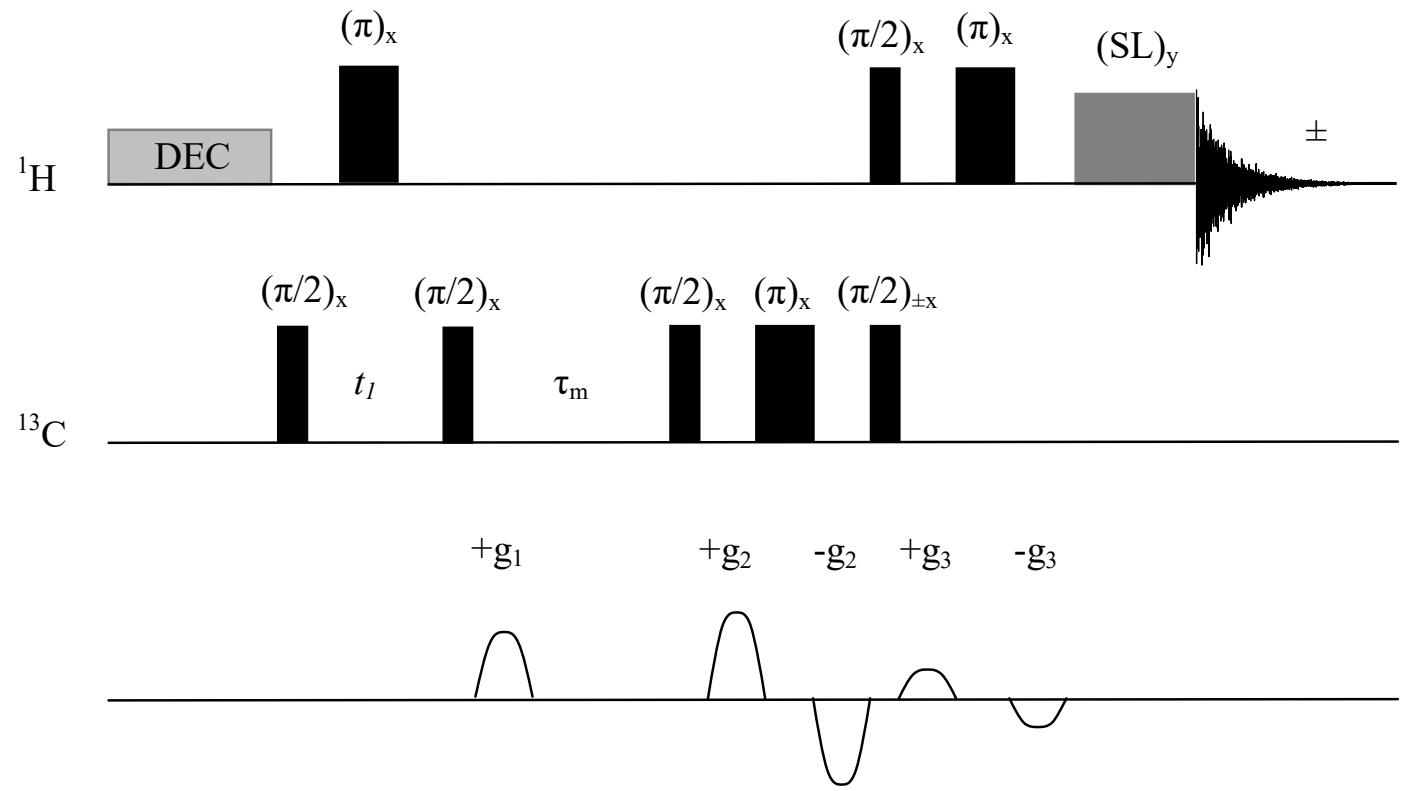

figure 1 


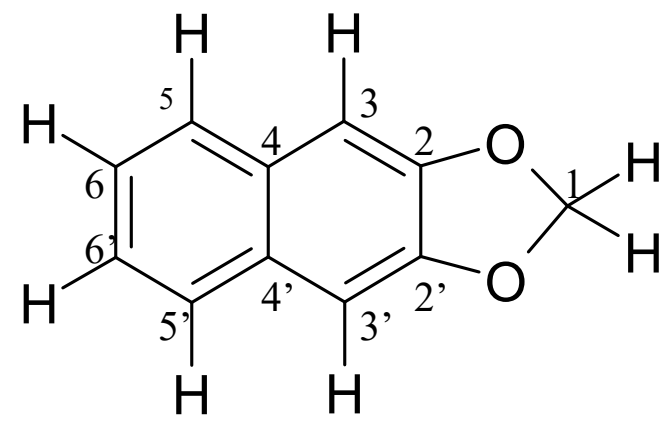

figure 2 


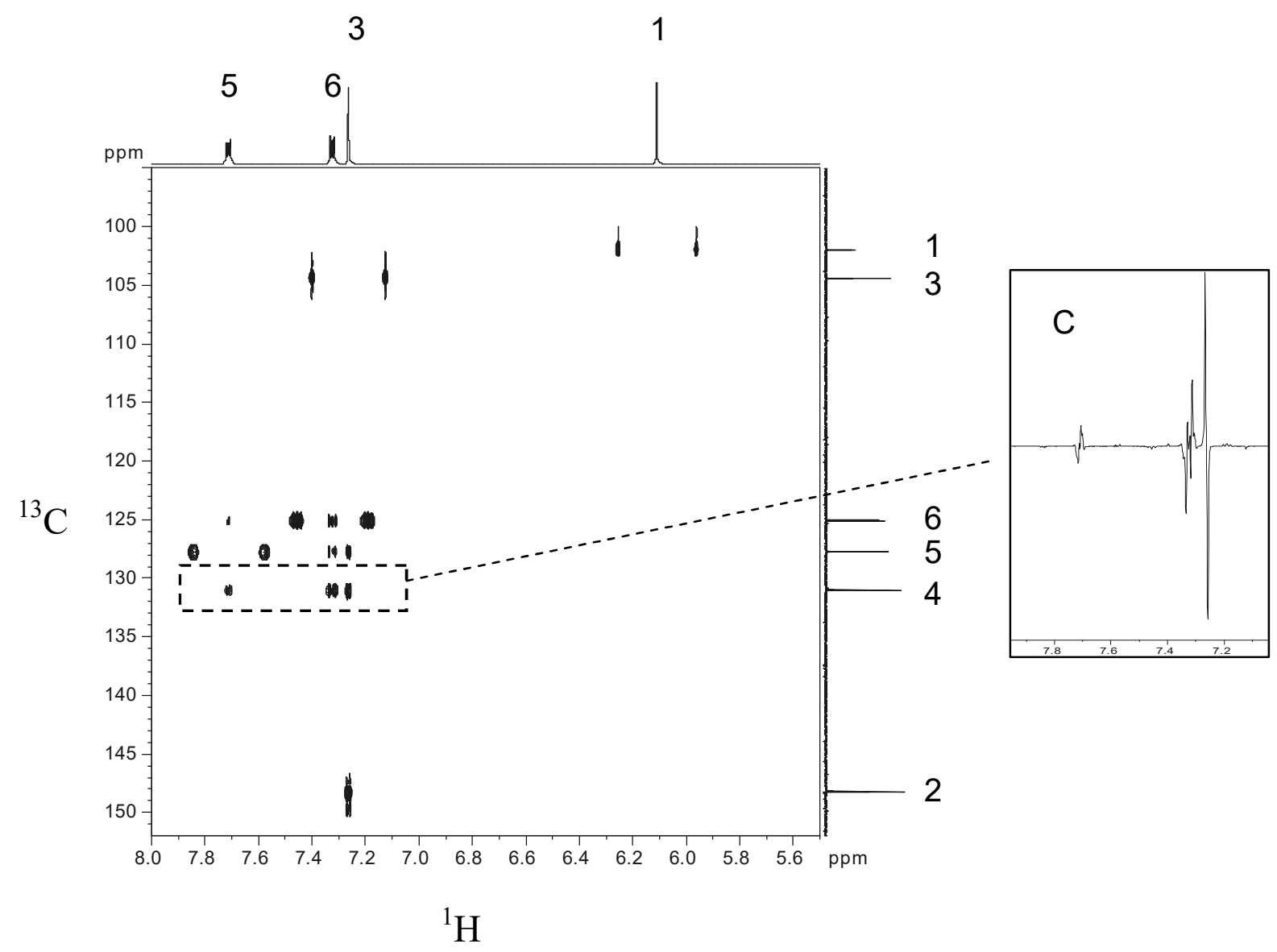

figure 3 


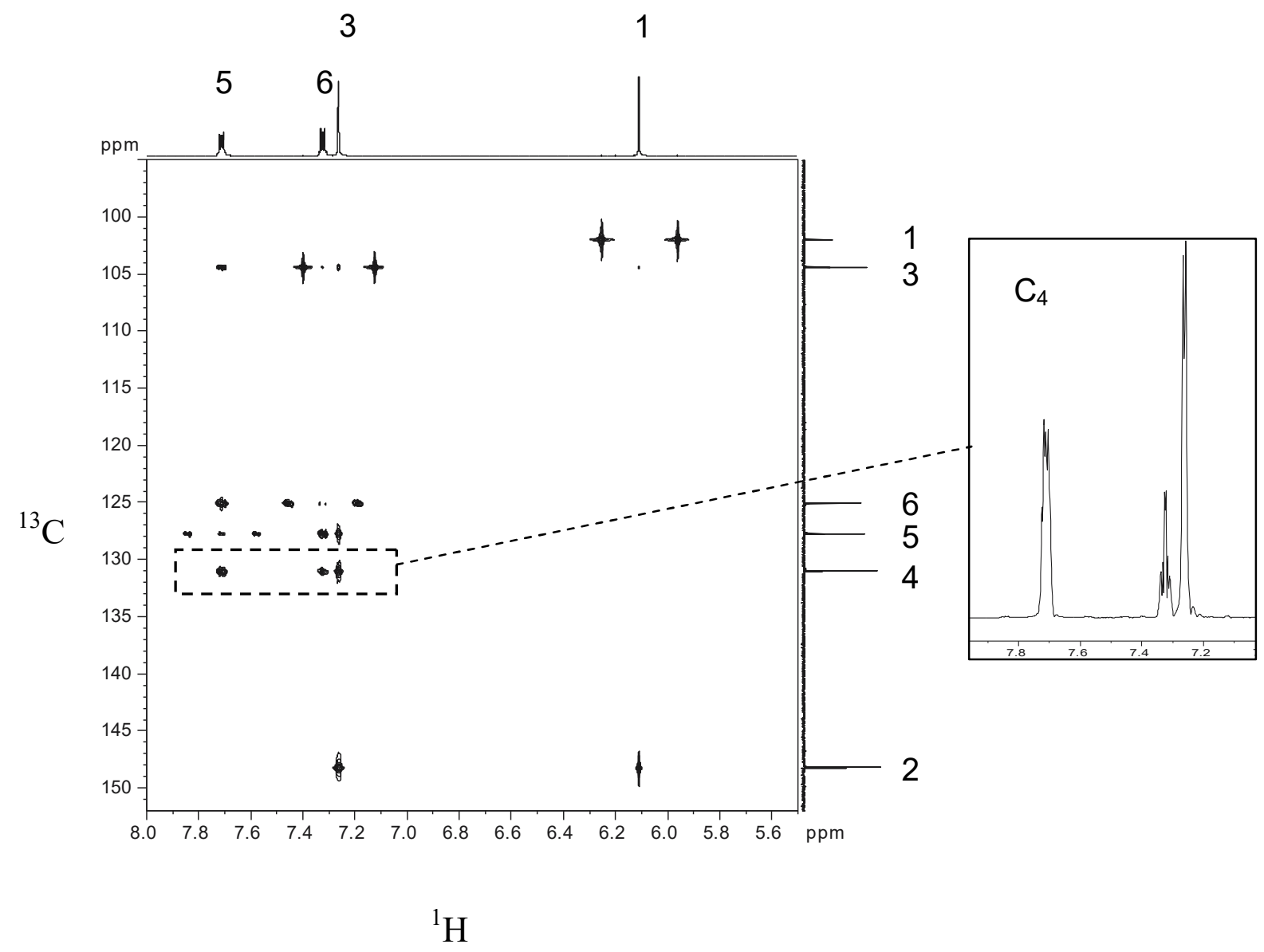

figure 4 